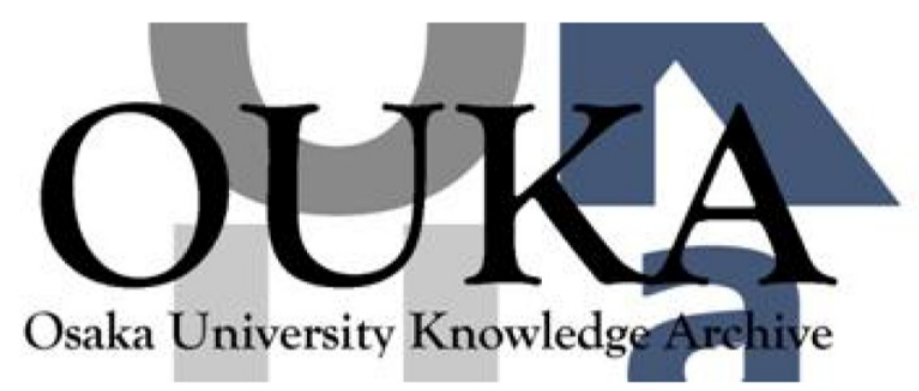

\begin{tabular}{|c|l|}
\hline Title & Position-Selected Molecular Ruler \\
\hline Author(s) & $\begin{array}{l}\text { Tanaka, Hirofumi; Anderson, Mary E. ; Horn, Mark } \\
\text { W. et al. }\end{array}$ \\
\hline Citation & $\begin{array}{l}\text { Japanese Journal of Applied Physics. 43(7B) } \\
\text { p. L950-p. L953 }\end{array}$ \\
\hline Issue Date & $2004-07$ \\
\hline oaire:version & AM \\
\hline URL & https://hdl. handle. net/11094/3231 \\
\hline rights & \\
\hline Note & \\
\hline
\end{tabular}

Osaka University Knowledge Archive : OUKA

https://ir. Library. osaka-u. ac. jp/

Osaka University 


\title{
Position-Selected Molecular Ruler
}

\author{
Hirofumi Tanaka*, Mary E. Anderson, Mark W. Horn ${ }^{1}$ and Paul S. Weiss ${ }^{\dagger}$ \\ Departments of Chemistry and Physics, The Pennsylvania State University, \\ 104 Davy Lab., University Park, PA 16802, USA. \\ ${ }^{1}$ Department of Engineering Science and Mechanics, The Pennsylvania State University, \\ University Park, PA 16802, USA. \\ * E-mail address: htanaka@ims.ac.jp \\ Present address: Center for Molecular Scale Nanoscience, Institute for Molecular Science, \\ 5-1 Higashiyama, Myodaiji, Okazaki 444-8787, Japan. \\ ${ }^{\dagger}$ Corresponding author. E-mail address: stm@psu.edu
}

Keywords: [molecular ruler, self-assembled monolayer, mercaptohexadecanoic acid, position select, electron irradiation, bottom up nanotechnology]

The molecular ruler method allows the precise control of the gap between a parent gold structure and a deposited daughter structure using a conveniently grown self-assembled molecular multilayer as a lithographic mask. However, we cannot choose a position where the gap should be placed, since the ruler attaches to all exposed gold surfaces. In this work, a convenient method of selecting the position of nanogaps by further patterning the molecular multilayer using low-energy electron beam irradiation and piranha etchant is described. 
The development of a convenient technique to fabricate precise nanostructures in large quantity is required for many types of application, such as electric devices, micromachines and biological equipment, since the "top-down" approach like electron lithography will reach its physical limit soon. Accordingly, it is urgently required to develop “bottom-up” techniques such as molecular self-assembly ${ }^{1,2}$ in order to achieve this goal.

Recently, many researchers have tried to create nanogaps between electrodes to measure the electronic properties of a single molecule or several molecules. For example, shadow evaporation $^{3}$, break junction ${ }^{4,5,6}$ and e-beam lithography ${ }^{7}$ have been investigated for this purpose. One of the most convenient approaches coupling the top-down and bottom-up approaches was developed as the molecular ruler method. $8,9,10,11$ This is based on the use of compact self-assembled molecular multilayers ${ }^{12}$ grown on parent gold structures as a convenient resist. Gap width is precisely controlled by the length of the molecular ruler and the number of monolayers deposited on the top of parent structures, for example, prepared by conventional lithography techniques. One of the advantages of this method is that both sides of the gap are vertically parallel and the desired gap sides can be formed precisely. Another advantage is the position of the gap can be controlled. Multilayers are grown by the successive sequential deposition of monolayers of $\alpha, \omega$-mercaptoalkanoic acid $\left(\mathrm{HS}(\mathrm{CH} 2)_{\mathrm{x}} \mathrm{COOH}\right)$ and $\mathrm{Cu}^{2+}$ ions, until the desired thickness is achieved. This molecular multilayered film covers the lithographically defined "parent” gold structures, forming a liftoff mask for the subsequently deposited metal layer. Then, the molecular resist is lifted off leaving behind the newly created daughter structures and the lithographically defined parent structures, as shown in Fig 1. However, the self-assembly method cannot determine the position of the parent structure, and the molecular ruler grows on all of them. This characteristic is a huge drawback for the application of this method in the preparation of complicated structures such as integrated circuits. In this work, we describe a procedure for further patterning of molecular ruler multilayers using low-energy electron beam irradiation and piranha etchant. In this way, we were able to prepare nanogap structures only in selected areas, imparting a fine position control to the molecular ruler method.

The method is schematized in Fig. 2. Starting gold parent structures were prepared by patterning a resist layer by the conventional photolithography and deposition of a 10-nm-thick Cr adhesion layer and a 40-nm-thick gold layer on a silicon wafer with a thermally grown $\mathrm{SiO}_{2}$ layer. In order to check the results of this method, gold was deposited $30 \mathrm{~nm}$ narrower 
than the Cr adhesion layer by the shadow deposition method. This substrate was cleaned with an ACT935 remover (Air Products and Chemicals, Inc.), rinsed thoroughly with deionized (DI) water and then immersed for $40 \mathrm{~min}$ in a $1 \mathrm{mM}$ solution of 16-mercapto-hexadecanoic acid (MHDA) in ethanol. Then, the substrate was rinsed with ethanol and dipped into a $1 \mathrm{mM}$ copper (II) perchlorate in the same solvent for $10 \mathrm{~min}$ and rinsed with DI water before depositing the next MHDA layer. This procedure was repeated 17 times in order to grow a 39nm-thick molecular ruler multilayer. ${ }^{13}$ Then, some selected portions of the molecular selfassembled layer were irradiated for 30 min with an electron beam at $1 \mathrm{kV}$, using an FE-SEM, and dipped in a piranha solution (Nanostrip; $\mathrm{H}_{2} \mathrm{SO}_{4}+\mathrm{H}_{2} \mathrm{O}_{2}$ ). In this way, only the molecular ruler multilayer in the nonirradiated area was removed, which allowed the control of the positions of nanogaps. This was confirmed by the deposition of a $\mathrm{Cr}$ layer and lifting off with ACT935 in order to obtain the subsequent "daughter" structures. This new method will be called the "position-selected molecular ruler (PS-MR)" method and the resulting gaps the “position-selected gaps (PS-Gs)” hereafter.

An arrangement of the observed sample is shown in Fig. 3(a). The circled area is observed by field-emission scanning electron microscopy (FE-SEM). FE-SEM images are shown in Figs. 3 (b)-(d). Three distinct sections are visible in Fig.3 (b). The bright bottom area corresponds to the nonirradiated area, from which the molecular ruler multilayer was removed with piranha solution, exposing the parent gold surface. The upper-left triangle shows the substrate's $\mathrm{SiO}_{2}$ surface and the upper-right rougher area corresponds to the irradiated molecular ruler multilayer that withstood the piranha solution and the nanostrip remover. As can be seen, the irradiated area of the gold structure shown on the left remained covered by the multilayer film, while it was completely removed from the nonirradiated section.

A Cr layer was evaporated on the previous sample, and then the molecular ruler was lifted off with an ACT 935 photoresist stripper and washed for 1 min with DI water in a sonicator. The FE-SEM image of a sample, prepared with 17 layers of MHDA/ $\mathrm{Cu}^{2+}$ (estimated thickness $=39 \mathrm{~nm}^{13}$ ), after the remaining processes to obtain the daughter structures is shown in Figs. 3(c) and (d). The darker area on the left corresponds to the daughter structure (D) of Cr, while the bottom right and upper-right parts correspond to the parent gold structure covered with the Cr layer (P/D) and the parent gold structure (P), respectively. Furthermore, a more or less well-defined gap with a width of approximately $30-50 \mathrm{~nm}$ is visible only in between the 
parent and daughter structures. This width is smaller than the thickness of the molecular ruler caused by the hydrocarbon stacking on them.

At this point, it is important to discuss why the irradiated multilayer molecular ruler becomes resistant to the piranha solution, making its patterning possible. A graphitic carbon layer is being formed on its top during the electron beam irradiation, due to the decomposition of hydrocarbon contaminants present on the surface of substrates in the vacuum chamber. ${ }^{14}$ The thickness of the carbonaceous deposition depends on the condition in the vacuum chamber, and the thickness should increase with irradiation time, although the average thickness measured in terms of height change by AFM was $11 \pm 3 \mathrm{~nm}$ in this sample, whose size allows us to estimate the lateral expansion of the molecular ruler. The hydrocarbon may chemically react with the molecular ruler to become an inert phase because nothing is disturbed to form the daughter structure on the exposed $\mathrm{SiO}_{2}$ substrate. This chemically inert carbonaceous layer should be protecting the underlying of the molecular ruler layer from the attack of the piranha solution. In fact, no carbonaceous impurities were found after lift-off of the PS-MR with the photoresist remover, supporting this assumption and ruling out the possibility of its complete decomposition. Furthermore, the decrease in gap width suggests that a combined mechanism should be operating. In other words, some carbonaceous deposition from hydrocarbon contaminants should be occurring concomitant with the deposition of carbon from contaminants present around the substrate or in the vacuum chamber. For the next step, gap size should be controlled more precisely to improve this fabrication method in order to apply this method for industrial use. Nanogaps with controlled width and position were successfully obtained, indicating that this strategy may be a widely used method for patterning molecular films for other applications.

In summary, we suggest a convenient procedure for further patterning of multilayered molecular films using low-energy electron beam irradiation and piranha solution, imparting position control to the molecular ruler method. We successfully controlled the position of a 40 $\mathrm{nm}$ nanogap using this simple and convenient molecular ruler method. The preparation of more complicated structures, such as nanotransistors or nanocircuits, has been realized using this method. Nanogaps with controlled width and position were successfully obtained, indicating that this strategy may be a useful method for patterning molecular films for other applications. 
This work has been funded by the Air Force Office of Scientific Research, Army Research Office, Defense Advanced Research Projects Agency, National Science Foundation, National Institute of Standards \& Technology, and Office of Naval Research, USA. Components of this work were conducted at the Penn State node of the NSF-funded National Nanotechnology Infrastructure Network. The authors thank Prof. Haiwon Lee of the Department of Chemistry, Hanyang University, Korea for fruitful discussions.

\section{Figure captions}

Fig.1 Schematic procedure of the molecular ruler method. ${ }^{8}$ (a) "Parent" structure was prepared on a substrate. The gold parent pattern is $30 \mathrm{~nm}$ thick and is on a 10-nmthick Cr buffer. (b) The molecular ruler was grown on the gold parent structure. (c) Daughter metal was deposited on the substrate (d) The molecular ruler was removed using a photoresist stripper. The gap between the parent and daughter structure is formed by the molecular ruler precisely.

Fig.2; Schematic procedure of PS-MR. (a) The "parent” structure is prepared on a substrate. The parent pattern is made of gold and is on a Cr buffer. The Cr buffer is $50 \mathrm{~nm}$ wider than the gold in order to observe results conveniently. The thickness of the Cr buffer is $10 \mathrm{~nm}$ and that of the gold is $40 \mathrm{~nm}$. (b) The molecular ruler was grown on the gold parent structure. (c) The molecular ruler is partially irradiated by an electron beam to cover the molecular ruler with hydrocarbons. (d) The sample is immersed in piranha etchant (Nanostrip). (f) Position-selected gaps are obtained between the parent gold and daughter Cr structures.

Fig.3; (a) Schematic of the sample during electron irradiation. PS-MR sample was observed by FE-SEM around the circled area. FE-SEM images of (b) Position-selected molecular ruler and (c) Position-selected gap between parent and daughter structures. Gaps are obtained between the parent and daughter structures only where the molecular ruler was present. (d) Gap created by PS-MR of another sample. Electron beam irradiated the sample 30 min to obtain the PS-MR. 


\section{References}

$1 \quad$ Y. N. Xia and G. M. Whitesides, Angew Chem Int Edit 37 (1998) 551.

2 R. D. Piner, J. Zhu, F. Xu, S. H. Hong and C. A. Mirkin, Science 283 (1999) 661.

3 Y. Naitoh, K. Tsukagoshi, K. Murata and W. Mizutani, e-J. Surf. Sci. Nanotech. 1 (2003) 41.

4 J. Park, A. N. Pasupathy, J. I. Goldsmith, C. Chang, Y. Yaish, J. R. Petta, M. Rinkoski, J. P. Sethna, H. D. Abrun • P. L. McEuen, and D. C. Ralph, Nature 417 (2002) 722.

$5 \quad$ W. Liang, M. P. Shores, M. Bockrath, J. R. Long and H. Park, Nature 417 (2002) 725.

6 M. A. Reed, C. Zhou, C. J. Muller, T. P. Burgin and J. M. Tour, Science 278 (1997) 252.

$7 \quad$ K. Araki, H. Endo, H. Tanaka and T. Ogawa, Jpn. J. Appl. Phys. 43 (2004) L634.

$8 \quad$ A. Hatzor and P. S. Weiss, Science 291 (2001) 1019.

9 M. E. Anderson, R. K. Smith, Z. J. Donhauser, A. Hatzor, P. A. Lewis, L. P. Tan, H. Tanaka, M. W. Horn and P. S. Weiss, J Vac Sci Technol B 20 (2002) 2739.

10 M. E. Anderson, L. P. Tan, H. Tanaka, M. Mihok, H. Lee, M. W. Horn and P. S. Weiss, J Vac Sci Technol B 21 (2003) 3116.

11 M. E. Anderson, L. P. Tan, M. Mihok, H. Tanaka, M. W. Horn, G. S. McCarty and P. S. Weiss, submitted for publication.

12 A. Ulman: An Introduction to Ultrathin Organic Filem (Academic Press, San Diego, 1991).

13 H. Tanaka, H. Lee, M. E. Anderson, L. P. Tan, M. Mihok, T. K. Ahn, S. M. Kim, J. D. Monnell and P. S. Weiss, in preparation for publication.

14 T. C. Isabell, P. E. Fischione, C. O'Keefe, M. U. Guruz and V. P. Dravid, Microsc Microanal 5 (1999) 126. 
(a)

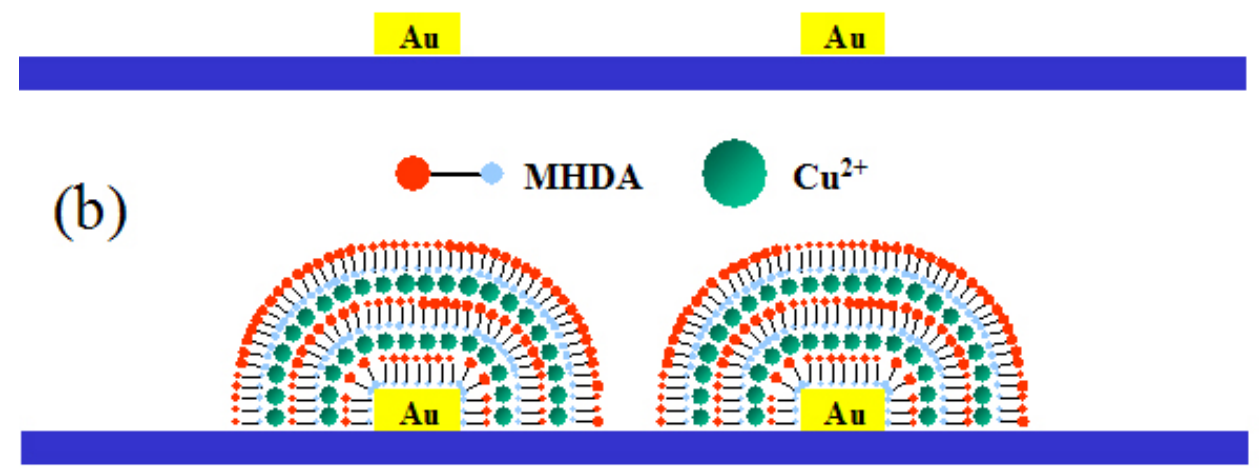

(c)

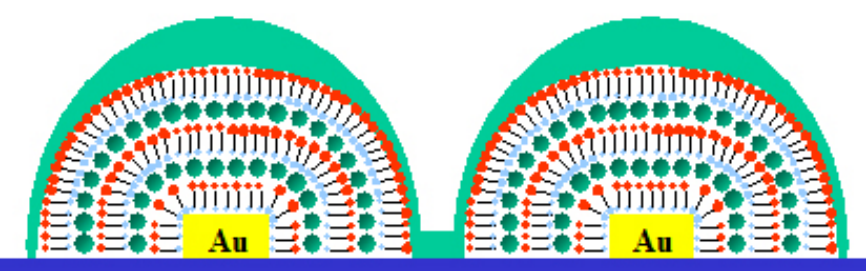

(d)

Fig. 1; H. Tanaka et al. 
(a)

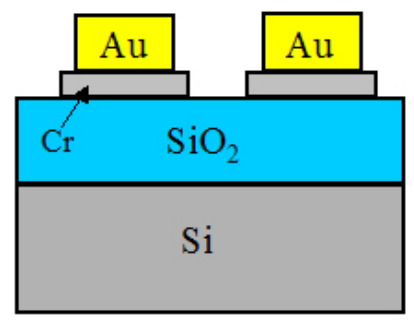

(d)

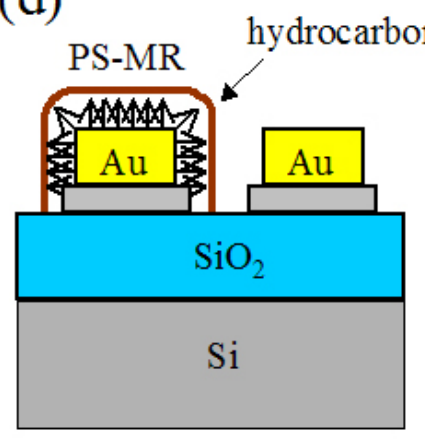

(b)

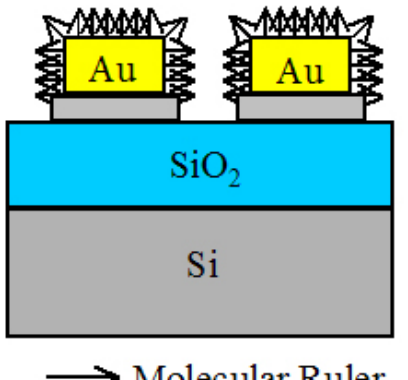

(e)

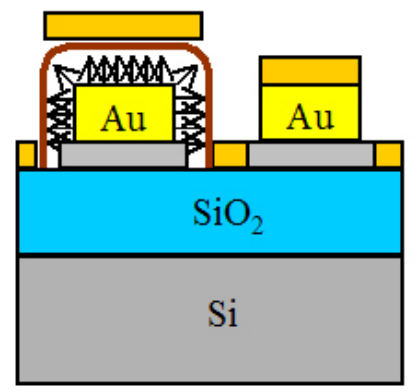

(c) e-beam

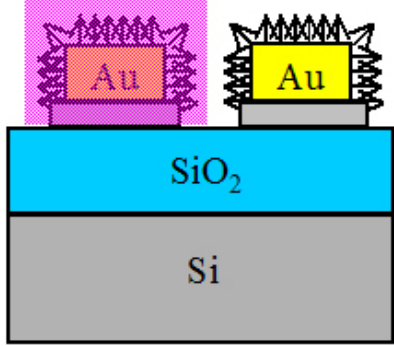

(f)

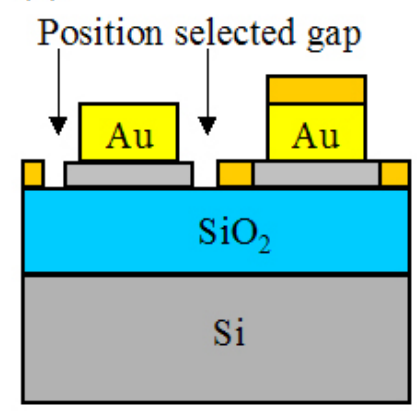

Fig. 2; H. Tanaka et al. 

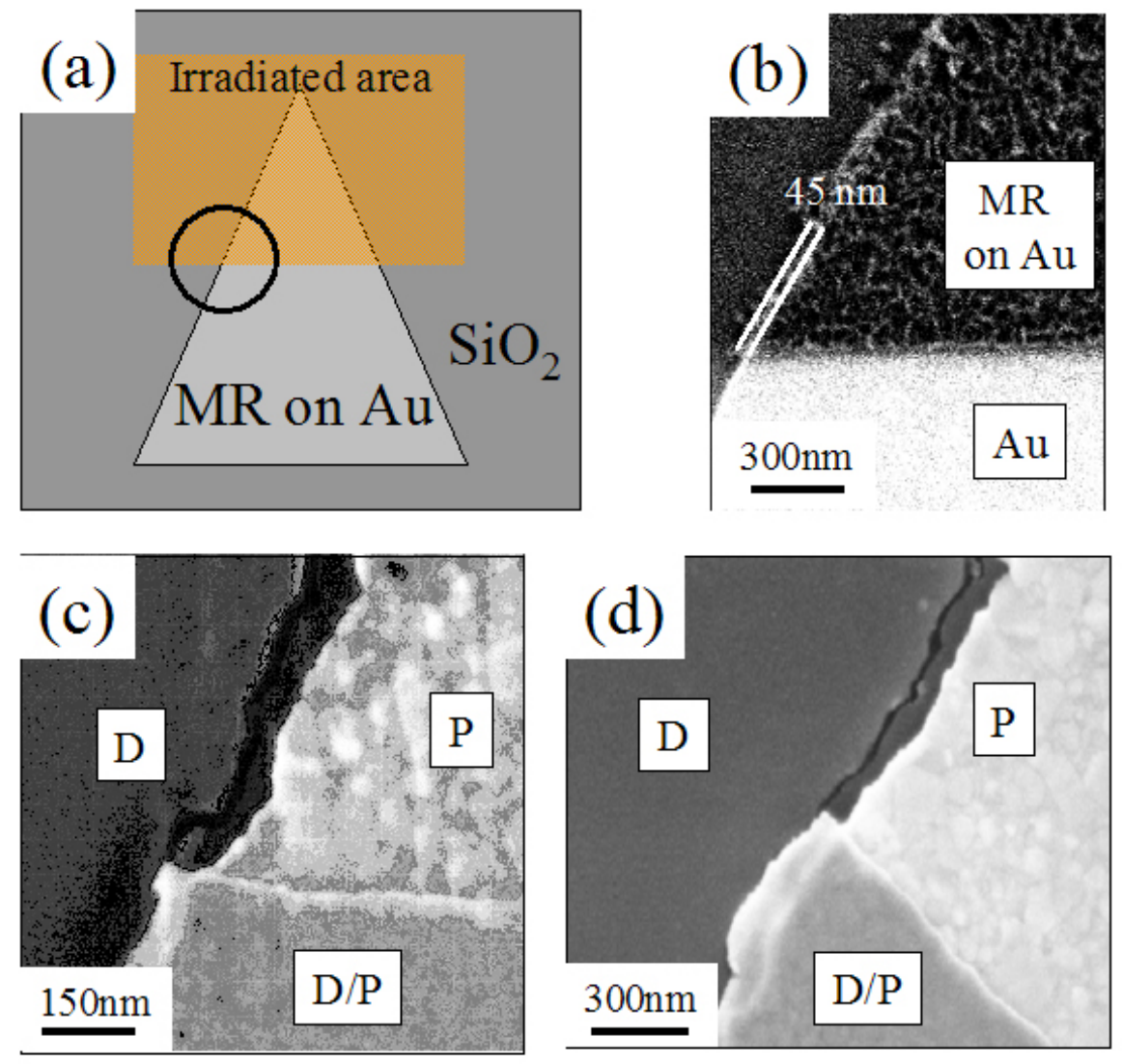

Fig. 3; H. Tanaka et al. 